\title{
Cincuenta años de trabajo duro: trabajando en los (¿yermos?) viñedos de la Administración pública *
}

\section{Bert A. Rockman **}

El propósito de este trabajo es examinar las evoluciones en el campo de la Administración pública durante los cincuenta años transcurridos desde la formación de IPSA. Este artículo no pretende ser un examen global de todos los cambios en la Administración pública y desde luego no es un tratamiento exhaustivo de toda la literatura relevante. Mi intención es destacar algunos de los trabajos dentro de la Administración pública, concebidos ampliamente -quizás tan ampliamente que muchos de los que faenan con esfuerzo en los viñedos de la. Administración pública no reconocerán como trabajos sobre la Administración pública algunos sobre los que yo me centraré. Esto, desde luego, refleja el tema de las «dos culturas» al cual alude Joonathan BENDOR (1994) para distinguir la investigación básica y la aplicada en el campo de la burocracia y de la Administración pública.

Mi camino comienza con los fundamentos microanalíticos de la elección en un contexto de teorías de caracterización neoinstitucionalista centradas, por una parte, en ideas psicológicas y antropológicas sobre las instituciones y, por otra, en la economía política de las instituciones. Comienzo donde hay mucha teoría aunque no muchos datos. Me muevo a continuación a la sociología política de las elites administrativas y luego a los estudios del funcionamiento organizativo donde hay muchos datos pero relativamente poca teoría. Finalmente, trato las teorías prescriptivas de la gestión para el sector público y las encuentro, en general, soprendentemente deficientes en cuanto a su estado científico. Son, frecuentemente, el producto de modas que del conocimiento. Desde esa perspectiva, las teorías sobre la estructura y organización adecuada del gobierno tienden a ser, si se me permite apoyarme en Herbert SIMON, parte de la tradición proverbial de la Administración.

Como cuestión general, es inevitable la conclusión de que hay una notable falta de conexión entre distintos tipos de trabajos englobados bajo los estudios de Administración pública y la burocracia. En segundo lugar, como se ha señalado, hay una tendencia, de un lado, a que existan repuestas empíricas a preguntas que no se sitúan dentro de teorías fuertes, y, de otro, a la existencia de teorías fuertes para las cuales no se dispone de pruebas científicas. Quizás, nada de esto es extraño o idiosincrásico, para el estudio de la Administración pública, pero hay algunas razones por las cuales sí podría ser así.

Primero, más que en la mayor parte de los campos, la Administración pública se halla dividida entre teorías positivas y normativas (aunque aquellos que se ocupan de las primeras con frecuencia tratan de encontrar una etiqueta diferente para caracterizarse a sí mismos (véase BENDOR, 1994) y entre una orientación de ciencia básica y una orientación pragmática. Estas orientaciones característica producen tradiciones de trabajo muy diferentes, frecuentemente a distintos niveles de análisis, y que se dirigen claramente a diferentes audiencias. Es bastante raro encontrar trabajos de una tradición que sean citados en el trabajo dentro de otra tradición a menos que se hiciese con propósitos de burla.

En segundo lugar, la Administración pública —y en realidad cualquier teoría de gestión y organización- está cargada de 
prescripciones o juicios previos sobre la naturaleza humana, sistemas de incentivos, capacidades humanas e, incluso, el significado de racionalidad. Y mucha parte de la teoría de la gestión también está cargada con juicios previos o presunciones sobre la naturaleza del gobierno (lo que constituye un «buen» gobierno) y con ideas sobre las diferencias y similitudes entre las actividades de los sectores público y privado. Estos temas se relacionan muy profundamente con las cuestiones más básicas sobre el papel adecuado del mismo sector público. Y, con frecuencia, tiene más que ver con juicios previos que con hechos comprobables. El campo de la Administración pública es, por tanto, intensamente normativo y, a la vez, está regido por juicios previos sobre la naturaleza humana y sobre el poder y sus designios.

El campo de la Administración pública está regido por temas fundamentales del poder y sus intenciones en el nivel macro y por creencias subyacentes sobre modelos de naturaleza humana en el nivel micro. ¿Hasta qué punto, por ejemplo, tenemos tendencia a ser emprendedores y buscar oportunidades e información? Los modelos organizativos de optimización implican que realmente buscaremos oportunidades cuando se nos dé la libertad para hacerlo. Alternativamente, chasta qué punto estamos limitados por nuestra naturaleza o por los costes de un ambiente puramente mercantil? La economía institucional a fin de cuentas se desarrolló poniendo énfasis en los costes de transacción inherentes a un ambiente de puro mercado y a la necesidad consiguiente de estructura (COASE, 1937; AlChIAN, 1950; ALChIAN y DemstZ, 1972).

¿Hasta qué punto, por ejemplo, y, precisamente, cómo debemos hacer responsables a los funcionarios? ¿Debemos usar formas o instrumentos basados en el mercado o basados en normas? ¿Cuánta discrecionalidad y qué tipo de discrecionalidad es la apropiada? ¿Qué significa el desempeño? Tales preguntas y otras reflejan ideas diferentes sobre cómo deben organizarse las personas, a qué incentivos responderán, e incluso, sobre lo que esperamos que consiga una organización. Una de las ironías de la reforma de gestión, incluyendo el énfasis en la organización basada en el mercado, es que, muy frecuentemente, se impone por la autoridad, incluso de forma autoritaria. La consecuencia de esto es que los procesos políticos normales interfieren, no solamente, con la expectativa de conseguir la reforma en la gestión, sino también con la misma gestión.

Donald KETTL (1993) a este respecto presenta muy adecuadamente una distinción a partir del contexto estadounidense entre el pensamiento hamiltoniano (jerárquico y estable) y el pensamiento madisoniano (pluralístico y no estable) ${ }^{1}$. Él observa dos escuelas diferentes de pensamiento que han estructurado el. campo de la Administración pública. Los subcampos más aplicados de la Administración pública y la gestión ponen el énfasis en el modo hamiltoniano (de pensamiento jerárquico) precisamente porque éstos se centran en la instrumentación eficaz para el logro de objetivos ${ }^{2}$. Esta instrumentación eficaz, desde luego, requiere objetivos conocidos y estables. Obviamente, las ideas tradicionales de la racionalidad organizativa y de gestión basadas en la cadena de mando reflejan la línea de pensamiento hamiltoniana. La distinción tradicional entre Administración o gestión, por una parte, y políticas, por otra, todavía prevalece en este contexto. KETTL advierte, sin embargo, una línea distinta del trabajo, de naturaleza más teórica, que está conformada por las ideas madisonianas. Esto incluye a la literatura sobre política burocrática, asimetrías de directoragente y competencia entre las agencias y entre directores.

La perspicaz distinción de KETTL es oportunamente mencionada, de manera diferente, por Jonathan BENDOR (1994) en otro análisis del campo de la Administración/Burocracia Pública. Creo que esta distinción puede ser valorada de la siguiente forma. Es probable que uno prefiera los puntos de vista hamiltonianos si:

1. lo que busca es poner en práctica un sistema de gestión;

2. lo que busca es ejecutar un sistema analítico;

3. lo que cree es que los juicios de los expertos y de los profesionales del gobierno deben prevalecer dentro de las líneas generales de política.

Alternativamente, uno preferiría las creencias o juicios madisonianos si:

1. lo que cree es que los equilibrios de política son inherentemente inestables;

2. lo que cree es que no hay consenso de expertos o de funcionarios excepto hasta el punto que puedan estar basados en autointereses organizativos o una cultura organizativa;

3. lo que cree es que en los juicios políticos, aunque sean distintos que los juicios de los expertos, deben ser evaluados por lo menos de la misma forma.

Creo que estos puntos de vista dan forma a mucho del recubrimiento normativo que rodea al trabajo hecho en la Administración pública y ayuda a explicar, como han notado otras personas, alguna de las diferencias entre la Administración pública y la gestión como profesiones prácticas y estudiosas y el trabajo teórico hecho por los científicos, los expertos en ciencia política y otros científicos sociales sobre la burocracia (BENDOR, 1994; MOE, 1994).

Otra distinción que creo que es de gran importancia y a las que ya he aludido, es la que hay entre la limitación de la racionalidad y las teorías de maximización de la utilidad. La racionalidad limitada, dicho de manera simple, es una teoría basada sobre las limitaciones cognitivas de la humanidad y las consecuencias que ello tiene para el comportamiento de las organizaciones. Las teorías basadas en la maximización de la utilidad suponen que los actores tienen una agenda de preferencias con estructuración de las elecciones transitivas y actua- 
rán para llevar adelante sus intereses de manera consecuente con ello. Estos asertos se encuentran en el núcleo de las teorías económicas de la burocracia y de la elección racional. Desde comienzos relativamente rudimentarios, este trabajo ha incorporado condiciones cada vez más sutiles e institucionales en los modelos, poniendo énfasis, por tanto, en estrategias de control y evasión entre las agencias y los supervisores y los usos de limitaciones legales e institucionales (MCNOLLGAST, 1990; MOE, 1990).

El trabajo clásico de Herbert Simon, El Comportamiento Administrativo, se publicó por primera vez en 1947, justamente hace más de cincuenta años. Puesto que su publicación y el período que consideramos coinciden más o menos y puesto que el libro es un hito en el estudio de la Administración y las organizaciones, empezaré con las distinciones microanalíticas que impulsan el trabajo de SIMON y el trabajo subsiguiente que amplía y desarrolla la tradición de la racionalidad limitada. A continuación trataré de desarrollar la lógica de los modelos de elección racional y a donde ha llevado este trabajo. No trato de decir que mi análisis agota la literatura ni tampoco se debe suponer que he intentado ser extenso más que ilustrativo.

A partir de juicios microscópicos sobre cómo se comporta la gente, paso a los aspectos empíricos del funcionamiento de las personas y de las organizaciones. Aquí las teorías son inducidas más que deducidas y son, con toda seguridad, construidas de manera menos rigurosa. Parecen conectar muy difícilmente con los fundamentos microanalíticos. A continuación, analizo los sistemas organizativos y de gestión como prescripciones y concluyo haciendo notar que la Administración pública es un campo muy fragmentado cuyos componentes científicos y prescriptivos, con frecuencia, parecen estar notablemente desconectados uno de otro.

\section{Fundamentos microanalíticos}

\section{Racionalidad limitada}

En 1947, se publicó El Comportamiento Administrativo de Herbert SIMON. Este libro, que era, originalmente, la tesis doctoral de Simon en la Universidad de Chicago, representó una importante señal para el campo de la Administración pública poniendo en cuestión las paneceas de la gestión clásica que habían sido desarrolladas por anteriores analistas del proceso de gestión (GuLICK, 1937). Las panaceas clásicas consideran posible la existencia de racionalidad en la gestión arriba-abajo, la coherencia y globalidad de la toma de decisiones y el carácter discreto de la elección. Aunque los campos empíricos flore- cientes de la sociología y psicología industrial ya estaban poniendo en cuestión las bases empíricas que subyacían a las premisas de racionalidad en la gestión, SIMON ofreció una teoría que se pulió y refinó posteriormente, que sugería que el campo de la Administración pública era prescriptivo pero no científico $y$, por tanto, frecuentemente equivocado (SIMON, 1958).

La esencia de la teoría de SimON es que los seres humanos son simplificadores y no complejificadores. Hay límites rígidos a nuestra capacidad de atención y a nuestros intereses para buscar respuestas completas en las ocasiones para las que satisfactoriamente sirven aproximaciones. Somos racionales pero de manera limitada. La limitación está definida, en parte, por los costes de oportunidad y por los costes de transacción. La necesidad de encontrar modos simples de operación conforma nuestro comportamiento porque los costes de hacerlo de otra manera son prohibitivos.

¿Cómo se relacionan estos juicios simples con la teoría de la organización? Para subrayar aquí, la esencia más que los detalles, las organizaciones como los individuos, desarrollan estrategias de resolución que reducen costes y hacen rutinario aquello que, de otra manera, tendría que pensarse desde el principio: Los procedimientos estandarizados de operación (SOP) son parte de este proceso de creación de rutinas, un proceso que WEBER también creía que era particularmente crucial en el fenómeno de la burocracia. La división de trabajo que es característico de las organizaciones complejas, y que WEBER creía que contribuía a su eficiencia, también puede ser una fuente de conflicto político dentro de las organizaciones y entre ellas, así como una fuente de tendencia al error en las organizaciones. Si los individuos no son proclives a buscar más allá de lo que necesitan -aunque haya que admitir que esto es un criterio vago-y si las organizaciones producen definiciones predecibles de los problemas, es evidente que los nuevos fenómenos se definirán de manera antigua, al menos hasta que la manera antigua pruebe ser claramente insuficiente para tratar con los nuevos fenómenos. Las organizaciones, por tanto, tienen tendencia a adaptarse lentamente porque hay poco incentivo para hacerlo de otra manera. La gente ve las cosas a través de las lentes de sus propias experiencias, y las organizaciones conforman sus experiencias. Cuanto más desarrollado está el esquema administrativo, más predecible es la respuesta (HALPERIN y KANTER, 1973). El posicionamiento burocrático y la política, por tanto, también pueden ser completamente predecibles.

Desde luego hay muchos más elementos procedentes del trabajo de SIMON y de aquellos asociados con la teoría de la racionalidad limitada. Uno de estos elementos es la importancia de las señales en un ambiente de atención limitada (MARCH, 1978). La noción de cambio de atención en ambientes cambiantes más que el cambio individual de preferencia es otro concepto que ayuda a explicar las modificaciones en las agendas (JONES, 1994a y 1994b) y permite establecer compatibilidad 
entre el supuesto de elección racional de las preferencias fijadas individualmente y el argumento de que la utilidad es un proceso en adaptación continua (COHEN y AXELROD, 1984).

La teoría de la racionalidad limitada nos sugiere, sobre todo, que, aunque las organizaciones pueden compensar algunas limitaciones humanas (tal como llevar a cabo tareas múltiples de manera simultánea, lo cual no podemos hacer individualmente) también pueden exagerar las condiciones humanas de intervalos limitados de atención y de seguir repertorios definidos por comportamientos anteriores. Además, el conflicto entre repertorios en competencia (distintas cosechas de política burocrática) es posible que se defina por jurisdicciones organizativas en competencia. Es posible que los procesos de búsqueda sean incompletos. Las tasas de error también pueden ser altas, puesto que la información se filtra entre las grietas organizativas (ANDER. SON, 1983).

Una posterior dicción a la teoría de la racionalidad limitada (aunque se ha puesto en tela de juicio por algunos críticos) es el llamado modelo de «cubo de basura» (garbage can). El modelo supone la existencia de corrientes de estímulos semiordenados que exigen atención organizativa. Pero dado que la capacidad de atención es limitada, los estímulos se fraccionan en problemas para ser procesados secuencialmente. Una crítica al modelo de cubo de basura (BENDOR, MOE y SCHOTTS, en preparación) mantiene que su conexión a la teoría de la racionalidad limitada es poco corriente, puesto que el cubo de basura es totalmente extraño a la teoría. Esta crítica también sugiere que el modelo subyacente y los procesos secuenciales de tomas de decisiones y de cuasirresolución no coinciden. Sin embargo, la base de la compatibilidad del modelo de cubo de basura con la teoría de racionalidad limitada se encuentra en la capacidad limitada que tienen las organizaciones para atender a corrientes de información de manera sinóptica y global. Desde este punto de vista, la teoría de la racionalidad limitada es fundamentalmente una teoría de procesamiento de la información en la cual la incertidumbre se reduce desde el punto de vista organizativo recurriendo a rutinas estandarizadas para reconocer y procesar los problemas. Cuando eso no puede ocurrir existe una condición de incertidumbre básica.

La contribución clave del modelo de racionalidad limitada es su enfoque sobre la escasez de atención, y la simplificación consiguiente de estímulos complejos convirtiéndolos en componentes descifrables moldeados por las rutinas y las definiciones de los problemas efectuadas por la organización. La adaptación gradual más que los mecanismos sinópticos o comprensivos de búsqueda es lo que define la forma en que buscamos respuestas a los problemas. En la famosa expresión de SIMON «los seres humanos intentan satisfice porque no tienen la inteligencia para maximize (aumentar al máximo)». Los fundamentos intelectuales de la racionalidad limitada se encuentran en la psicología cognitiva y van en sentido contrario a los de la economía clásica. Sus implicaciones son que el mundo se hace predecible por las rutinas y estructuras organizadas que imponemos sobre él (desde luego WEBER tenía una idea similar). Al mismo tiempo, las rutinas y estructuras inducen una tendencia al error cuando los nuevos estímulos se fuerzan para ser reconocidos a través de las viejas categorías o rutinas ${ }^{3}$. Empaquetar y atraer la atención son cruciales. La persistencia de rutinas en competencia en las organizaciones y la existencia de definiciones de problemas en competencia también dan lugar a posiciones competitivas que disminuyen el aspecto de control y coordinación del modelo weberiano. Una materia para debate es cómo se debería evaluar esta competencia, puesto que algunas teorías de la burocracia (weberianas) concluyen que la unidad de mando es central para la efectividad mientras que teorías de la burocracia basadas en el mercado defienden que la competencia entre secciones de la burocracia genera aumentos de la eficiencia (WoLF, 1999).

Las ideas de racionalidad limitada han llevado a un trabajo neoinstitucionalista liderado por MARCH y OLSEN (1983, 1989, 1995). En este trabajo las organizaciones no se convierten simplemente en un conjunto de reglas, sino más bien en un conjunto institucionalizado de normas. Podría decirse que las instituciones no coinciden con las organizaciones, sino con las normas institucionalizadas de lo que es el «carácter apropiado» del cual se ha investido a las organizaciones y en el cual se han socializado sus miembros. No es sorprendente que estas ideas se hayan originado parcialmente y parezcan más extendidas en Escandinavia donde la organización e integración normativa también están más extendidas. Es innecesario decir que puede ser frustrante científicamente saber con precisión qué es apropiado cómo, cuándo y dónde, en ausencia de una formulación explícita pero una implicación segura es que las instituciones no son simplemente estructuras; son principalmente culturas. Las culturas reducen el mundo a un conjunto de normas sobre lo que es o no es apropiado. En un mundo tal el conocimiento de lo qué es y no es apropiado limita las búsquedas.

Al final no está claro si las normas se derivan de las estructuras y de las tecnologías básicas que las impulsan o si actúan fundamentalmente para lubricar las relaciones entre estructuras que representan distintos intereses. La primera de las ideas sugiere que los intereses exclusivos (podría decirse funciones homogéneas de utilidad) están en la base de las normas, mientras la segunda idea sugiere que las normas mejoran el potencial para un choque (OLSEN, 1983). Puesto que no pueden coexistir fácilmente las dos nociones (exclusividad e integración) uno podría preguntarse ca qué nivel es, particularmente, apropiado el «carácter apropiado»? Este problema de la deducción de normas en presencia del pluralismo organizativo constituye un rompecabezas. $\mathrm{O}$ las estructuras pluralísticas producen diversas culturas institucionales (en virtud de la homogeneidad de cada 
una) y una cultura mediadora es creada exógenamente o, como se dice en la literatura clásica sobre el pluralismo americano, (Truman, 1951) producida a través de intereses superpuestos. ¿Puede la lógica del carácter apropiado, en palabras cualesquiera, tener la misma posibilidad de crear políticas burocráticas intensas para mejorarlo? De hecho, ¿cuál es la lógica precisa detrás del carácter apropiado?

\section{Elección racional}

El neoinstitucionalismo también es el producto de modelos de elección racional. A primera vista esto parece extraño, puesto que las típicas teorías de elección racional están basadas en la maximización de la utilidad en un ambiente que está exclusivamente conformado por las preferencias individuales. La conceptualización de SHEPSLE de un «equilibrio institucional» se deriva, sin embargo, de su noción de «instituciones de equilibrio» (1983, 1989). Se puede decir que un equilibrio institucional es el producto de las necesidades de los actores para crear reglas y normas que permitan conseguir una acción colectiva (en la cual, presumiblemente, cada actor tiene un papel) o impedirla (donde, de nuevo, cada actor puede tener un papel). SHEPSLE y WEINGST (1987), por ejemplo, explican la emergencia de normas de reciprocidad de comité en la Cámara de Representantes de los Estados Unidos como una función de búsqueda de formas de proteger los intereses individuales aumentándolos al mismo tiempo. Las normas y reglas que gobiernan la subordinación a competencias jurisdiccionales especializadas (diremos más sobre esto más tarde) proporcionan las condiciones de equilibrio que definen los movimientos posibles de los actores. Debe notarse que aunque el concepto de instituciones de equilibrio debería aplicarse, potencialmente, a cualquier conjunto de instituciones y en cualquier lugar, se centra en gran medida en el Congreso de los Estados Unidos —una institución que tiene reglas complicadas, con gran adorno, con existencia independiente y con grandes oportunidades para la autonomía individual- De hecho, una de sus Cámaras —el Senadotiene reglas que siguen promocionando la autonomía individual a expensas de la acción colectiva más de lo que lo hace la otra Cámara (the House). Es importante entender que mucho del apoyo del neoinstitucionalismo de la elección racional se encuentra en el contexto estadounidense por las peculiaridades de las instituciones políticas estadounidenses, especialmente las legislativas. Estas instituciones proporcionan tanto autonomía institucional como una independencia individual considerable. Es precisamente su independencia lo que permite las maniobras estratégicas.

En lo que se refiere a la Administración pública y a la burocracia, es natural, por tanto, que la literatura sobre la elección racional se centre de manera extensa en las relaciones del director-agente. Estas relaciones implican, entre otras cosas, reglas concernientes a los procedimientos de supervisión y a las condiciones (especialmente las que implican certidumbre e incertidumbre) bajo las cuales se pueden utilizar los procedimientos ex ante y ex post (MCCubBins, 1985; McCubBins, Noll y WeIN. GAST, 1990). También incluyen estrategias por medio de las cuales las agencias mejoran su autonomía a través de asimetrías de la información sobre sus directores implicados (BENDOR, TAY. LOR y VAN GAalen, 1987). Pero también puede incluir relaciones entre múltiples directores para crear condiciones colusorias o monopolistas/oligopolistas bajo las cuales las agencias y sus supervisores putativos fijan la oferta y el «precio» de los bienes públicos a un nivel mayor que su función de demanda subyacente (NISKANEN, 1971; FIORINA, 1978).

La mayor parte de esta literatura surge del acuerdo de separación de poderes del sistema de gobierno estadounidense bajo el cual es posible tener múltiples mandantes sobre la burocracia. Aunque una forma de gobierno dividido ha tenido lugar en otros sitios distintos de los Estados Unidos ${ }^{4}$, en ningún otro contexto es tan grande el potencial para competir sobre el control de la burocracia. Y son estas condiciones de conflicto probable entre varias personas con autoridad (implicadas en la separación de poderes sin tener en cuenta su división partidista real) las que dan lugar a varios modelos de dominio de uno u otro actor: la burocracia, la legislatura o el presidente.

Uno de los primeros trabajos que puso énfasis en el dominio burocrático fue La burocracia y el gobierno representativo de William NisKanen. La clave era que los departamentos proporcionarían bienes en exceso abasteciendo, por tanto, a los revisores monopolísticos (directores, especialmente los comités relevantes del Congreso encargados de las autorizaciones y de las subvenciones) y clientes cuya influencia sobre los revisores también sería, posiblemente, grande y cuya función de demanda para los bienes producidos por cualquier departamento específico era conmensurablemente grande. El resultado, de acuerdo con este breve análisis, era que la oferta de bienes públicos por los departamentos y su demanda pública subyacente no estaban alineados porque la oferta estaba sesgada hacia los grupos de clientela monopolística de los departamentos y hacia los comités del Congreso influenciados por estos grupos.

Aunque el modelo de NISKANEN coloca al departamento y a sus ventajas de información en el centro, su modelo, que se describe, con frecuencia, como de dominio burocrático, se puede caracterizar con igual validez como un modelo de colusión entre el departamento, sus patrocinadores, y supervisores bajo condiciones en las cuales los supervisores y patrocinadores se superponen de manera significativa. Presumiblemente solamente el presidente (para quien NISKANEN trabajó en una ocasión) se encuentra fuera del círculo y, por tanto, es más posible que represente un interés público. 
En el centro del modelo de NISKANEN se encuentra la creencia de que los burócratas individuales quieren maximizar sus propias ganancias personales consiguiendo recursos más sustanciales para sus agencias. La falta de conexión lógica, y especialmente empírica, entre la ventaja individual y los recursos de la agencia se ha documentado de manera completa causando que NiSKANEN se retracte de alguno de los elementos claves de su formulación original (BLAIS y DiON, 1991; NISKANEN, 1991).

Alternativamente, el argumento de que la legislatura controla el comportamiento de los departamentos (dominio legislativo) es la interpretación más popular de la relación director-agente (FIORINa, 1978; McNollgast, Weingast y Moran, 1983) en el contexto estadounidense. Aunque tenga formas variadas, el argumento básico se enuncia más o menos así: hay comités dominantes con jurisdicciones fijas cuyos miembros son atraídos a esas jurisdicciones por los beneficios esperados que posiblemente obtendrán. Por tanto, el comité (frecuentemente concretado como un comité en un cuerpo unicameral) mantiene los resortes y empuja a los departamentos a proporcionar bienes distributivos que redundan en el beneficio político personal de los miembros del comité. El hilo que conecta los intereses de los legisladores y el control del departamento es la legendaria conexión electoral. En el grado en que los departamentos pueden proporcionar bienes distributivos para los electorados del legislador 0 , alternativamente, en el grado en que los departamentos pueden imponer costes en esos electorados, los legisladores tienen poderosos incentivos para forzar a los departamentos a un comportamiento más acomodaticio. Un sistema de obligaciones legislativas funciona cuando los comités tienen funciones de utilidad homogéneas, es decir, los miembros tienen intereses del electorado similares que les conducen a querer estar en comités específicos, y los mismos comités discrepan respecto a la tendencia central de su cámara. El juicio subyacente es que las jurisdicciones de los comités son fijas y no se superponen.

Lo que varía para la mayoría del comité es la información y la certidumbre que a su vez influye en las estrategias utilizadas por los legisladores para controlar a las agencias, por ejemplo, si buscarán controles ex ante (típicamente bajo condiciones de certeza) o controles ex post (típicamente bajo condiciones de incertidumbre), o si el control aplicado varía con las propiedades del área de política de que se trate (BAwN, 1997). La literatura de dominio legislativo, sin embargo, sigue siendo llamativamente idiosincrásica, es decir, se aplica al poderoso, complejo e individualista sistema legislativo que prevalece en los Estados Unidos con representación territorial y altos niveles de visibilidad personal de los candidatos. Algunas de sus suposiciones, especialmente sobre exclusividad del control del comité sobre la política, han sido revisadas cuidadosamente en su aspecto empírico (BAumgarTner, Jones y MacLeod, 2000).
Sin embargo, un enfoque diferente del problema de competencia entre mandantes (¿quién ejercita el poder?) es el que reclama que los presidentes tienen la última palabra (MoE, $1985,1989,1999)$ sobre la burocracia en virtud de que 1) no tienen un problema de acción colectiva, es decir, hay un presidente con un pensamiento, pero 535 legisladores con intereses y pensamientos diversos, distribuidos en dos cámaras muy diferentes, y 2) retienen la capacidad para iniciar acciones que después colocan a otros actores, especialmente, el legislativo, a la defensiva. Hay otra evidencia que sugiere que cuando los presidentes generan fuertes señales también consiguen que las agencias respondan (WoOd y WaTERMAN, 1991). Las señales, sin embargo, pueden definirse, de manera adaptativa, por las agencias y las personas con autoridad, puesto que cada uno responde a la incertidumbre (CARPENTER, 1996). Pero el problema real es encontrar las condiciones bajo las cuales cada conjunto de directores es probable que ejerza control y las circunstancias bajo las cuales los departamentos evaden el control o ejercitan la autonomía. La colusión, con frecuencia, puede tomar una apariencia superficial de conflicto (ABERBACH, 1990) cuando los intereses favorables a los programas de una agencia pueden evitar investigaciones más críticas de las agencias al embarcarse en otras menos críticas.

El enfoque de los modelos institucionales de elección racional tiende a situarse en el problema de la competencia entre personas con autoridad, el ocultamiento, las asimetrías de información, y las estrategias de control bajo condiciones de incertidumbre. Lo esencial de todo esto es quién ejercita el poder (o no necesita ejercerlo porque las agencias operan por deferencia a las necesidades de algún conjunto de personas con autoridad). Hay una gran cantidad de teorización sobre esto, pero, irónicamente, no demasiada que llegue bien fuera de Estados Unidos. En principio, desde luego, podría hacerse y el trabajo de LaVer y Shepsle (1994), McCubBins y Noble (1995) sugiere que es verdaderamente posible. Pero Moe y Caldwell (1994), por ejemplo, hacen notar que las diferencias entre los sistemas parlamentarios y los sistemas presidenciales (de poderes separados) también da lugar a enormes diferencias en las posibilidades de control coherente. Creo que MOE y CALDWELL exageran este caso particular; sin embargo, hay también alguna verdad en ello. Es verdad que los legislativos tienen menos capacidad de controlar las agencias a través de incentivos individualistas y estructuras legislativas fragmentadas. Pero, desde luego, los partidos podrían tener esas capacidades y las usarán para beneficiar sus expectativas políticas beneficiando a sus electores. Es innecesario decir que ese argumento también se puede aplicar a los presidentes en el sistema estadounidense que siendo tan políticos como los legisladores tienen electorados políticos particulares a los que necesitan pagar. Sin embargo, es la increíble complicación del sistema estadounidense lo que convierte al paradigma del director-agente y los temas de dele- 
gación y firma en una industria virtual para los expertos en ciencia política en los EE.UU.

Entonces, irónicamente mucha de la teoría institucional sobre la elección racional está limitada por un sistema idiosincrásico. Sistemas estructurados de manera más simple tendrán todavía problemas de control que no son producto, simplemente, del dispositivo institucional. Por ejemplo, el comportamiento evasivo es posible casi en cualquier sitio y las preferencias incompletas por parte de los superiores también pueden dar cuenta de las tendencias de los departamentos a seguir sus propios caminos, al menos hasta que eso entre en conflicto abierto con los intereses de sus supuestos superiores. En otras palabras, hay límites inherentes al control organizativo y éstos son, en parte, una función del procesamiento de la información en un mundo de intervalos limitados de atención. Tales temas nos traen de vuelta a otros antiguos, pero pertinentes, sobre la gestión, tal como el de los intervalos apropiados de control.

\section{En resumen}

Las bases microanalíticas de la literatura sobre burocracia o la Administración pública tienden a tratar a la misma Administración sólo de manera tangencial. Al poner énfasis en el procesamiento de la información los teóricos de la racionalidad limitada llaman la atención sobre las limitaciones, tendencias al error y miopía de los comportamientos organizativos que se desprenden de la necesidad de reducir la complejidad informativa. Por otra parte, los teóricos de la elección racional institucional ponen énfasis en la estructura existente en la formación de los incentivos, en los costes de información, asimetrías de la información, incluso, en el desarrollo de normas. Los teóricos de la elección racional institucional tienden a ver el mundo en términos de intereses en competencia estructurados por las instituciones y en donde distintos conjuntos de información y costes de acción colectiva se asocian con la posición estratégica de los actores dentro del contexto institucional. Se centra en la competencia para el control de las agencias, el logro monopolístico de control, la incapacidad de control (dominio del departamento) o estrategias recíprocas de influencia (Arnold, 1980; Krause, 1999).

La perspectiva de racionalidad limitada pone el énfasis en los procesos organizativos como forma de tratar con la complejidad de la información. Estos procesos, sin embargo, producen inevitablemente sus propias patologías. La predictabilidad viene del lado organizativo de la ecuación y la impredictabilidad viene del ambiente. La forma en que las dos se encuentran, la incertidumbre ambiental y las estructuras y rutinas organizativas, es la base del modelo de «cubo de la basura» originado por los teóricos inspirados en la racionalidad limitada (Cohen, March y Olsen, 1972; March, Olsen y Cohen, 1976).
Las premisas que hay tras de los modelos de racionalidad limitada son inherentemente pesimistas sobre las capacidades de las organizaciones para resolver problemas, excepto que sean problemas de procesos organizativos. Sin embargo, las organizaciones se enfrentan a los problemas y casi los resuelven encontrando un acuerdo momentáneo que posiblemente se puede convertir en uno de más duración. Las premisas tras de los modelos de elección racional también se pueden considerar, en último término, como pesimistas en el sentido que, o los departamentos tienen autonomía para dictar sus propios términos, disminuyendo, de esta manera, su posibilidad de control democrático, o están sujetos al control preponderante de un tipo de supervisor con exclusión de otros, produciendo, por tanto, una forma asimétrica de respuesta a la política.

Mientras que las teorías de nivel individual proporcionan una base sustancial para el trabajo sobre la burocracia, las teorías resultan ser difíciles de probar directamente. Se deben hacer unas cuantas consideraciones auxiliares y es especialmente difícil evaluar con precisión cómo se puede distinguir la racionalidad limitada de la variedad sin límites, puesto que las teorías de elección racional se han quedado incluidas en un contexto institucional que en sí mismo limita las elecciones.

De las condiciones poderosas desde el punto de vista teórico, pero débiles desde el punto de vista empírico que tiene lugar desde el micronivel, pasaré, ahora, a considerar el trabajo en un nivel más macroscópico con una base de datos más rica, pero con una teoría menos potente que lo sustente.

\section{La sociología política de las elites administrativas}

\section{Composición y carreras de los mandarines}

¿Quiénes son los burócratas? En especial, ¿quiénes son los que se encuentran en la cima del sistema? Los juicios detrás de esta pregunta (qué, en otras palabras, hace que tenga consecuencias) son: 1) la burocracia es importante en sí misma (y, autónoma, hasta cierto punto); 2) la burocracia es un camino hacia otras posiciones de poder y prestigio en la sociedad (el fenómeno de pantouflage); 3) la composición de la burocracia del nivel superior refleja su conexión o desconexión con la sociedad civil, y 4) la composición de la burocracia del nivel superior revela las prioridades y necesidades cambiantes de los gobiernos o de un gobierno determinado.

Los estudios sobre la composición de los funcionarios superiores (y también sus ministros) son numerosos. Esto puede 
tener que ver con la posibilidad de que éstos sean los datos que están más disponibles para los estudiosos. Además, ésta es una materia en la que parece haber un cierto número de estudios transnacionales (DOGAN, 1975; KuBOTA, 1969; KOH, 1989; Campbell y Szablowski, 1979; Page y Wright, 1999; Sulweiman, 1974; Aberbach, Putnam y Rockman, 1981; AberBACH y ROCKMAN, 2000, entre otros). No sorprende que se preste mucha atención a las elites burocráticas en contextos como el francés y el japonés donde se considera que la burocracia tiene un poder considerable. Verdaderamente, en los lugares en los que la burocracia tiene la reputación de mantener un gran nivel de autonomía, los estudios sugieren que los canales de reclutamiento tienden a ser exclusivos y estrechos. También hay pruebas, especialmente en Francia y en Japón, que cuanto más prestigiosa es la burocracia más probable es que constituya una plataforma de lanzamiento hacia otros sectores elitistas de la sociedad, tales como los negocios de las empresas o las elites políticas (BirnBaum, 1982; SulEiman, 1978).

Hasta cierto punto, estos estudios se relacionan con los cuatro juicios indicados al principio de esta sección. Tratan, especialmente de forma un poco más directa, de la segunda y tercera cuestión, es decir, de la interconexión entre la elite burocrática y otros sectores elitistas y del grado relativo de conexión o desconexión con la sociedad civil. Hablan del primer y cuarto punto utilizando más la inferencia. Sin embargo, e incluso, en las cuestiones en las cuales los datos ajustados pueden dar una explicación más directa, las implicaciones de comportamiento con frecuencia están implícitas o son conjeturas más que resultados de la investigación. Esto se debe, en parte, a que las implicaciones o las conjeturas no están siempre claras. Por ejemplo, ¿cuál sería la diferencia en el comportamiento de una burocracia más representativa con respeto a una menos representativa? En cuestiones tales, se hacen más conjeturas que demostraciones (ABERBACH y ROCKMAN, 2000: 47-51). Y, de hecho, no siempre es claro el significado de la representatividad. ¿Es posible, por ejemplo, que los canales exclusivos de reclutamiento basados en el mérito, puedan realmente producir un cuerpo de burócratas más representativo socialmente que los basados, principalmente, en lazos de clase o familiares o, incluso, de patronazgo?

La primera consideración sobre el poder de la burocracia lleva, esencialmente, a las teorías clásicas de la elite. Los estudios de Suleiman $(1974,1978)$ de los grandes cuerpos franceses, por ejemplo, implica que son difícilmente unitarios monolíticamente en sus preferencias. Más bien se infiere que la elite administrativa francesa está preocupada, sobre todo, con la capacidad de controlar los medios mediante los cuales se reproduce como elite. Ésta es la definición clásica de gremio, de manera similar a la que definen los profesores de universidad que, de manera parecida, tienen preferencias e intereses diversos, pero que, sin embargo, están unidos en gran medida en su deseo de controlar los medios mediante los cuales ellos, como grupo, se reproducen y reciben las credenciales. Cuando se considera que la burocracia tiene un status alto y es un centro importante de poder, muy probablemente crece la preocupación sobre los medios mediante los que se incorpora allí la gente convirtiéndose en un tema de democracia e igualdad. Cuando no se considera a la burocracia de esa manera, lo más probable es que la preocupación tenga que ver con la eficiencia o com. petencia.

La cuarta consideración tiene que ver con los posibles cambios, a lo largo del tiempo, en los tipos de competencia que prevalecen en los distintos gobiernos así como diferentes énfasis en la competencia a través de los diferentes sistemas nacionales. Por ejemplo, ¿se fijan las burocracias gubernamentales en personas con una formación general o en «tecnócratas»? Indudablemente cuanta mayor formación general tiene el individuo, más énfasis se pone en completar el discurso propio, es decir, ser listo y cultivado, y ágil, desde el punto de vista intelectual y estilístico. Una burocracia que se construye alrededor de este concepto, como el caso británico, por ejemplo, se concentrará posiblemente de manera significativa en aquéllos con una educación adecuada y con una formación escolar que pone énfasis en la educación tradicional. Esto también significará, probablemente, que existe un canal relativamente estrecho a través del cual la elite administrativa llega a ocupar sus posiciones y en donde muy pocas prestigiosas universidades dirigen a sus licenciados hacia la función pública en un porcentaje desproporcionado. Por el contrario, una burocracia tecnocrática pone gran énfasis en destrezas específicas, aunque, claramente aquéllos que tiene mas probabilidades de llegar a la cima tienen también otras características y talentos, particularmente conocimiento respecto a la política burocrática. Uno también puede darse cuenta de los cambios que tienen lugar dentro de un sistema observando los datos sobre su composición y las trayectorias profesionales. Sin embargo, lo que signifiquen esos cambios es objeto de conjeturas más amplias y, con frecuencia, inciertas.

Paradójicamente en los casos en que tenemos muchos datos nuestras teorías están especificadas menos claramente y son más tentativas. Hay, a fin de cuentas, una teoría formulada claramente que especifique con precisión cómo trabaja la burocracia bajo distintas características referentes a su composición y a las trayectorias profesionales? ¿Sabemos realmente cuáles son los impactos de distintas composiciones, por ejemplo, que la burocracia sea más o menos representativa (y, cंcon respecto a qué características?) o que se tengan licenciados de universidades más o menos prestigiosas? Lo que sí sabemos, desde luego, es que por lo general las características básicas no explican el comportamiento demasiado bien entre las elites en el nivel individual y, especialmente, en situaciones en las que los papeles están relativamente limitados. Sin embargo, las cues- 
tiones más importantes pueden ser cómo se percibe la burocracia en la sociedad. Pero el hecho es que no sabemos específicamente qué se podría inferir del estudio de los perfiles de formación general de los individuos que constituyen la elite de la función pública. Típicamente se hacen más cosas con estos datos de lo que es razonable.

\section{Actitudes y comportamiento de los mandarines}

Aunque cada vez el número sea mayor, hay menos estudios que examinan, a través de entrevistas, las actitudes y, hasta cierto punto, el comportamiento de las elites administrativas. Muchos de los trabajos que se citaron anteriormente sobre la composición de los funcionarios públicos de alto nivel también tienen datos que se han recogido sobre las actitudes y el comportamiento (véase también CAMPBELL, 1983; CAMPBELL y WiLSON, 1995). Las clases de cuestiones que pueden ser capaces de contestar son de la clase siguiente:

1. ¿Hasta qué punto las definiciones organizativas o de papeles correlacionan con (o realmente estructuran causalmente) las actitudes?

2. ¿Hasta qué punto están los funcionarios públicos en sintonía política o ideológica con sus superiores políticos o con el público en general?

3. ¿Hasta qué punto los funcionarios públicos y sus superiores (ministros, etc.), confían o se valoran entre sí?

4. ¿Hasta qué punto están los funcionarios satisfechos o insatisfechos con sus trabajos o con las condiciones políticas o de otra clase que afectan a sus responsabilidades?

5. ¿Hasta qué punto los diferentes actores relevantes para la Administración (ministros frente a funcionarios o legisladores frente a funcionarios, por ejemplo), piensan en realidad de manera diferente?

6. ¿Hasta qué punto podemos inferir pautas de toma de decisiones e influencia a partir de los comportamientos observados?

7. ¿Hasta qué punto son los funcionarios públicos sensibles o escépticos ante las reformas administrativas?

8. ¿Hasta qué punto se está transformando la cultura de la burocracia?

Aunque las cuestiones anteriores son empíricas y, ciertamente, no son inherentemente teóricas hay varias de ellas que tienen implicaciones teóricas más amplias especialmente en lo que respecta a cuestiones como la sensibilidad de los funcionarios públicos y las funciones predictivas del papel o la formación básica en lo que concierne a sus actitudes y comportamiento. Igualmente las pautas de relación entre los elegidos y los seleccionados se pueden explorar a través de la indagación empírica de las actitudes de la elite.
Las preferencias expresadas, sin embargo, son a veces despachadas, especialmente por los teóricos de la elección racional. La expresión de las preferencias (en contraposición de la revelación de las referencias bajo limitaciones de mercado) se ve, con frecuencia, como sin coste (y, por tanto, inválida) y ateórica. Es parte de la división entre lo sociológico y lo económico (BARRY, 1978; MoE, 1994). En un lado de la división (el sociológico) hay respuestas específicas a cuestiones teóricas formuladas vagamente. En el otro (el económico) hay, con frecuencia, respuestas teóricas solamente a las preguntas empíricas. Hasta el punto de que los datos actitudinales sean considerados válidos - pueden ayudar a contestar preguntas empíricas específicas y aclarar el valor de varias proposiciones-. Sin embargo, si la definición propia de teoría se refiere exclusivamente a lo axiomático, es poco probable que los métodos empíricos de investigación satisfarán esa definición. Pero, en ultimo término, la ciencia funciona mediante la comprobación empírica de proposiciones y no simplemente mediante su formulación o afirmación, cualquiera que sea la elegancia con que se haga (GREEN y SHAPIRO, 1994). Además, el producto de la evaluación empírica puede producir interpretaciones en competencia y resultados aparentemente diversos que están afectados por la variabilidad en la medida, las técnicas analíticas, la operacionalización y la especificación cuyo análisis se considera normalmente que constituye la ciencia normal.

Otra parte de la literatura sociológica sobre las organizaciones que está con frecuencia sujeta a acusaciones similares de hiper-empirismo es la literatura sobre funcionamiento organizativo, que revisaré, brevemente, a continuación.

\section{Función organizativa}

Hay una extensa literatura sobre función organizativa. Recomienda una revisión de Administrative Science Quarterly, entre las revistas de investigación, así como de un gran número de revistas de gestión, estudios de casos, e incluso, revistas de psicología industrial. El problema es que no hay una única teoría de efectividad organizativa, una condición que lleva a los teóricos de la elección racional a proclamar las deficiencias inherentes al estudio de la efectividad organizativa sin una teoría potente (MoE, 1994). Desde luego, han habido teorías potentes en el pasado $\multimap$ por lo menos teorías mantenidas enérgicamente- incluyendo las teorías sobre «relaciones humanas». Lo que sucede simplemente es que no han salido tan bien como las curas singulares o incluso significativas de los problemas organizativos. Vale la pena citar aquí con cierta extensión al autor de un estudio que compara diferentes tipos de organizaciones: 
Los gestores que puedan haber leído este libro por la promesa implicada en el título esperando encontrar información clara de que un único marco organizativo o estilo de liderazgo está relacionado con la efectividad se habrán desencantado. Las prescripciones aisladas para conseguir la efectividad son como espejismos: deseables pero distantes, retrocediendo, y reales. Nuestra investigación muestra que varios estilos de liderazgo y estructura organizativa pueden ser efectivos. Es necesario apoyarse en los factores condicionantes... discutidos aquí para determinar las técnicas apropiadas y estructuras que deben ser usadas. La selección de la estructura de toma de decisiones apropiada está influenciada por la legitimación de las diversas estructuras alternativas, la proporción de profesionales en la plantilla, por el hecho de que exista o no una emergencia, por el grado de improvisación que implica el trabajo y por varias cosas más. De manera similar, el grado de estructura de la tarea y entre las tareas afecta a la manera en que otras características organizativas se relacionan con la efectividad. De esta manera hay normalmente varias formas de organizar y liderar que darán al final prácticamente los mismos niveles de efectividad (MотT, 1972: 184-185).

Es perfectamente evidente que tal complejidad (a la cual, con frecuencia, se la denomina mediante los términos incongruentes «teoría de la contingencia») es para aquellos que ya están convencidos de que hay una forma de tratar con la complejidad (MoE, 1994). Desde luego, hay siempre una manera de tratar con la complejidad. Pero otra cuestión es si es válida empíricamente, lo cual es, al final, la única comprobación científica de una proposición.

El hecho es que los meta-estudios de las propiedades organizativas y los estudios comparativos de un único proyecto tienden a proporcionar relativamente pocos resultados claros. Por ejemplo, un meta-análisis reciente del fenómeno de la privatización muestra lo complicados que son los resultados y su carácter condicional (HodGe, 1999). Estos resultados ambiguos pueden ser una función de una teoría pobre, de poner demasiados ingredientes en la olla, y de no retirarse un poco para contemplar la imagen general (MoE, 1993). Pero también puede deberse a que la teoría potente es potente, solamente, cuando no se prueba o es imposible probarla. En un artículo reciente y provocador Paul PIERSON nos recuerda que a pesar de las «nobles aspiraciones para desarrollar una ciencia de la política fundamentada en la parsimonia y la generalización y capaz de gran poder predictivo... la incapacidad para generar generalizaciones potentes que faciliten la predicción, sigue siendo un problema» (2000: 266). Un problema que, según indica, puede deberse al carácter del mismo mundo político.

\section{En resumen}

De esta manera hemos vuelto al punto inicial. Las teorías que comienzan en el nivel individual desarrollan claras lógicas a medida que se mueven hacia la acción colectiva y los fenómenos organizativos. Pero los resultados sobre la acción colectiva y los fenómenos organizativos pueden ser consistentes con más de una explicación. Y en cualquier caso, muchos de los datos relevantes para estas premisas son experimentales, cuasiexperimentales, o basados en casos. Alternativamente, en el nivel intermedio encontramos que tenemos más datos del mundo real de características cuantitativas sistemáticas, pero está menos claro qué teorías guían en la recolección de datos o en su análisis o, en otras palabras, comprendemos menos cuál es la pregunta (al menos la pregunta teórica) que corresponde a esa respuesta.

En este aspecto el estudio de la Administración difiere poco, probablemente, de otros campos de la ciencia política. Pero es diferente al menos de dos maneras. En primer lugar, las teorías de la organización dependen de las teorías de la naturaleza humana. ¿De qué somos capaces? ¿Qué haremos con la discrecionalidad o con las limitaciones de la jerarquía? ¿So. mos entes que maximizan la utilidad y buscan información $o$, por el contrario, somos seres de costumbres, altamente sensibilizados hacia los costes de las transacciones? En segundo lugar, ¿dependen de lo que sabemos las teorías prescriptivas sobre cómo se debe organizar? Pero lo que sabemos es necesariamente contingente y mucho depende de lo que hacen las organizaciones y de cómo lo hacen. Si una regla no vale para todos, ¿cuántas reglas necesitamos? ¿Cuántas condiciones diferentes dictan prescripciones diferentes? ¿Cómo se vende la complejidad y la condicionalidad cuando se exige simplicidad? $¿$ ¿Hay y puede haber un conjunto nuclear subyacente de conceptos detrás del funcionamiento organizativo? Y a esto también habría que añadir la cuestión poco delicada de équé es exactamente el funcionamiento? Verdaderamente, esta pregunta, que es especialmente prominente en la esfera pública, aunque no exclusiva de ella, está siendo debatida todavía, incluso, entre los más fervientes partidarios de la evaluación organizativa ${ }^{5}$.

Tales cuestiones nos llevan, naturalmente, a considerar las teorías de gestión que subyacen a las reformas en la estructura y organización del gobierno. ¿Cuáles son las bases empíricas de tales reformas? ¿Y en qué «modelos del hombre» están basadas? La primera respuesta a esa cuestión puede ser, desde luego, muy práctica y puede depender de quién esté auspiciando la reforma y, por tanto, de cuáles resulten ser sus intereses para la reforma. LigTH (1998), por ejemplo, indica que las reformas de los Estados Unidos auspiciadas por la legislatura se enfocan típicamente hacia temas de responsabilidad y de control más estricto, de tal manera que se limite la discrecionalidad del ejecutivo y, consecuentemente, la evasión de los esfuerzos legis- 
lativos para controlar la burocracia. Las reformas auspiciadas por el ejecutivo, sin embargo, tienden a estar basadas en el funcionamiento y se diseñan para evitar que el legislativo intervenga con éxito. En otras palabras, cada parte tiene intereses específicos en la reforma relacionados a su propia capacidad para controlar o influir en la burocracia.

Pero más allá de estas peculiaridades estructurales de la situación en los Estados Unidos y la competencia que resulta para controlar la burocracia, es necesario escrutar las bases teóricas y empíricas de las ideas de reforma. A fin de cuentas éste es el punto en el cual la prescripción se encuentra con la realidad. ¿Hasta qué punto son las prescripciones contrastadas con la realidad? ¿Hasta qué punto son consistentes, teóricamente? ¿Hasta qué punto son meras preferencias 0 , peor todavía, simples modas? Estas preguntas no pueden contestarse de manera definitiva aquí, ni, quizá, en otro sitio, pero se plantean en la sección siguiente haciendo énfasis en su complejidad subyacente.

\section{Introduciendo reformas organizativas y de gestión en el gobierno}

Las reformas en el sector público son consecuencia de una mezcla de motivos, siendo las ganancias políticas obviamente uno de ellos, cuando no el más importante. Como indican ABER. BACH y RoCKMAN, «todas [las reformas] cambian el equilibrio de intereses y valores» (2000: 13). Pero las reformas también son producto de otras cosas: la necesidad de considerar los cambios en el entorno del gobierno; la moda y el contagio; y la necesidad de parecer competente y, especialmente, eficiente en la gestión de gobierno. No hay duda de que las reformas del gobierno siguen estas dos indicaciones que están relacionadas entre sí. Una indicación es «icuál es el problema al cual da respuesta la reforma?». La segunda es «cicuáles son las soluciones que están de moda para estos problemas?».

En los años sesenta estaban de moda las soluciones generales analíticas para los problemas públicos, en parte porque la agenda de gobierno incluía muchos programas nuevos o en expansión. Los intelectuales de la política estaban muy de moda y la creencia de que el gobierno podía incorporar la analítica racional a sus gastos era muy fuerte. En este contexto se convirtió en un enfoque prominente la analítica basada en el presupuesto. La reforma ponía ahora menos énfasis en la estructura de gestión (un producto de los esfuerzos anteriores para racionalizar la Administración a través de dispositivos como el POSCORB) y más en la analítica de la toma de decisiones y el pensamiento estratégico de las políticas. Fue en este contexto en el que se introdujeron los sistemas de Programación, Planificación y Presupuestación [Program, Planning, Budgeting Systems (PPBS)] y que fueron obligatorios durante un tiempo en el nivel federal del sistema de los Estados Unidos. Como técnica, los PPBS se suponía que mantenían la presupuestación centrada en el panorama amplio de funcionamiento más que en las líneas de presupuestación per se. La lógica era analíticamente clara, aunque fuese amenazadora políticamente. Significaba que había una latitud inherentemente grande para el ejecutivo en lo que respecta a la presupuestación y el control de la política y, por tanto, amenazaba al electorado y a los intereses ligados a los programas en funcionamiento. Realmente, las reformas sinópticas del presupuesto han sido criticadas, generalmente, por su insensibilidad para con las personas implicadas y para con el orden creado por las políticas de interés de la clientela en los programas existentes (WILDASKY y HaMmond, 1965). Desde luego, ésta es la razón verdadera para la reforma del presupuesto. Se intentaba, precisamente, extirpar esos intereses y debilitar sus apoyos institucionales. Las reformas comprensivas del presupuesto fueron siempre y seguirán siendo una herramienta para debilitar los intereses que crea la política fragmentaria. La racionalización promueve el poder en el centro y desde la cima.

En resumen, ni los PPBS ni las técnicas presupuestarias sinópticas que les sucedieron, como el presupuesto en Base Cero [Zero-Bases Budgeting (ZBB)] que se acomodan a la estructura organizativa en la cual estaban situados los programas existentes. Un sistema fragmentado se resiste a una lógica de racionalidad de unificación política. Los sistemas exigieron más del gobierno de lo que era posible y, esencialmente, exigían la ausencia de presiones políticas para mantener un statu quo favorable o resistirse a la imposición de un statu quo menos favorable. La analítica de la política no murió con la disminución del énfasis cuando no el cambio de los sistemas comprensivos de tomas de decisiones presupuestarias. Pero la analítica de la política tomó un rumbo diferente y más tangible durante las Administraciones de Carter y, especialmente, de Reagan en los Estados Unidos, durante las cuales se aplicaron análisis coste-beneficios a las reglamentaciones propuestas por el gobierno con intención obvia de disminuir la actividad regulatoria.

El próximo cambio importante, sin embargo, se orientó, principalmente, hacia temas de gestión como parte de una tendencia internacional para introducir un Estado tradicionalmente menos burocrático y más austero. Las reformas que fueron introducidas principalmente en los años ochenta en países como Australia, Nueva Zelanda y el Reino Unido y que fueron incorporadas posteriormente por otros a lo largo de los años noventa se conocieron como Nueva Gestión Pública [New Public Management (NPM)] y en los Estados Unidos como la Reinvención del Gobierno (Reinventing Government). El síndrome de la 
NPM está compuesto de diferentes aspectos. Sin embargo, está esencialmente impulsada por la idea de que la microgestión desde arriba es una mala idea y de que «conducir» pero no «impulsar» es lo que se debe hacer desde arriba. Presume de potenciar a los burócratas de más bajo nivel proporcionándoles la discrecionalidad para enfrentarse a los retos. Superficialmente, el objetivo es alejarse del estilo científico de alta diferenciación de tareas preconizado por los teóricos de la gestión de principios del siglo xx. Al mismo tiempo, la NPM haría responsables a los administradores del funcionamiento, aunque existen ideas diferentes sobre cómo debería hacerse esto ${ }^{6}$.

Después de todo esto, el tenor general de la NPM tiende hacia una mayor flexibilidad administrativa, mayores respuestas a los usuarios de servicios, un cuadro más flexible de gestores, remplazando en algunos casos o coexistiendo con una función pública por oposición, un Estado más ligero con más contratación de sus actividades, una mayor privatización de algunos de sus bienes, y en general, más competencia entre los proveedores gubernamentales y privados, al mismo tiempo que se pone un mayor énfasis en el control del funcionamiento y la responsabilidad ex post. En resumen, el producto sería un Estado más flexible y con capacidad de adaptación que sería gestionado con un énfasis en los intermediarios, la toma de riesgos y el desarrollo de sistemas planos compatible con la era de la información más que con las formas jerárquicas antiguas (Os. BORNE y GAEBLER). Estaría orientado al cliente y sería accesible; sería competitivo y sus funciones estarían cada vez más privatizadas o transferidas al exterior.

Apostaría a que pocos de los gurúes de la gestión pública han consultado trabajos serios de las ciencias sociales que traten con las complejidades de la estructura organizativa, los sistemas de incentivos, y la cooperación dentro de organizaciones no jerárquicas (G. MILLER, 1992) o hayan hecho un inventario de lo que sabemos realmente sobre el funcionamiento gubernamental (T. Miller, 1998) o la privatización (HodGE, 1999. Si lo hubiesen hecho habría menos posibilidades de que hubiese mucho menos compromiso, casi religioso a una u otra vía. Tal como advierte un conjunto de analistas sobre las explicaciones del crecimiento del gobierno ( $\mathrm{y}$ también de las preferencias de formas de organización impulsadas por el mercado), «siempre está ahí la tentación de afirmar más de lo que tu "ciencia" puede justificar» (LARKEY, STOLP y WINER, 1984: 94).

La gran ironía, por tanto, es que a pesar de un desarrollo teórico considerable en el estudio de la Administración y la burocracia y a pesar de un crecimiento igualmente considerable en los estudios sobre el funcionamiento, las reglas para la reforma de la gestión (que desde luego son, fundamentalmente, mucho más que reforma de la gestión) están muy poco basadas en cualquiera de los dos. Se venden normalmente como panaceas. La complejidad y la sutileza son difíciles de vender, especialmente, cuando tienen que venderse a los políticos que, de hecho, pueden estar más interesados en lo que parece que funciona que en lo que funciona realmente.

\section{Unos cuantos pensamientos finales}

Por tanto, nos quedamos curiosamente con tres mundos de la Administración pública aparentemente independientes. Uno centrado en los fundamentos micro-analíticos, que es fuertemente teórico y menos claramente empírico; un segundo, centrado en estudios del funcionamiento del personal y de la organización, que es fuertemente empírico pero menos declaradamente teórico; y el tercero, que involucra al mundo de la praxis, que parece haberse impregnado poco, tanto de la literatura empírica como teórica. Resulta que tenemos un triángulo muy poco agradable, en el que cada lado tiene poco que ver con el otro. Lo que es todavía peor, las ideas para la reforma del gobierno se presentan, típicamente, con la convicción de los proponentes más que enfocarse con escepticismo científico. El marketing y la política dominan el entramado de la reforma.

En los más de cincuenta años desde que El Comportamiento Administrativo de SimON llegó a las librerías, el estudio de la Administración y la burocracia ha avanzado grandemente aunque sea de manera desequilibrada. Realmente nos hemos movido más allá de los tópicos sobre la Administración y tenemos muchos más datos sobre los sistemas administrativos y sus contextos de los que existían hace medio siglo. El viñedo de la Administración pública no ha sido estéril, pero el campo sufre realmente del problema de tener una multiplicidad de comunidades compactas que, raramente, se comunican entre sí. Además, en la práctica, las propuestas de reforma son, con frecuencia, el producto de buenos deseos o de marketing entusiasta más que de un análisis cuidadoso o de razones científicas. No es probable, por ejemplo, que en el mundo de la investigación de campo los analistas aplicados pudiesen ser tan informales con la base de conocimiento que se ha acumulado en su campo. Pero cuando se trata de la práctica de la gestión, no existe el mismo énfasis en el conocimiento acumulativo. La razón por la que esto es así es una cuestión que nos debe ocupar como campo de conocimiento. 
Traducción de Elisa Romero Sebastín.

* Comunicación preparada para el World Congress of the International Science Association, RC27.3, Developments in the Study of the Structure and Organization of Government Since 1949, Qubec City, Canada, 31 de julio al 6 de agosto de 2000.

** Universidad de Pittsburgh.

"Alexander Hamilton fue el primer Secretario del Tesoro de los Estados Unidos y fue uno de los tres autores de los artículos federalistas que precedieron a la convención institucional de 1787 en Filadelfia. Las preocupaciones de Hamilton eran tener un Estado federal fuerte y un poder ejecutivo fuerte también. Su filosofía se podría describir de la manera más adecuada como un «modernizador del Estado conservador». James Madison, el cuarto Presidente de los Estados Unidos, se considera como el principal arquitecto de la Constitución de los Estados Unidos (y fue también uno de los tres autores de los artículos federalistas). Su filosofía se caracterizaría más apropiadamente como «pluralismo institucional», poniendo el énfasis en lo apropiado de contrastar los intereses entre instituciones constituidas de manera diferente.

${ }^{12}$ Gary Miller y Thomas Hammond (1994) hacen notar que el criterio de eficiencia, sin embargo, es difícilmente obtenible de una forma neutra, por tanto, la política es más fundamental que la economía para proveer los bienes públicos.

${ }^{13}$ Sobre este punto particular BENDOR y HAMmOND (1992: 319) hacen notar que las rutinas organizativas permiten la elección y no la limitan simplemente. A partir de esto también concluye que las rutinas no siempre predicen el comportamiento.
${ }^{14}$ Más claramente en Francia que durante un período significativo de tiempo ha tenido un sistema de cohabitación en el cual el presidente y la mayoría parlamentaria y su gobierno son de diferentes tendencias políticas partidistas. Hay otros casos donde los nuevos procedimientos electorales hacen posible la creación de gobiernos divididos. Y, desde luego, se pueden considerar de manera parecida las coaliciones multipartidistas (LAVER y ShePSLE). Sin embargo, en ninguno de estos casos es la legislatura tan poderosa como en los Estados Unidos y en ninguno es tan grande la capacidad de influir en la Administración.

${ }^{15}$ Por ejemplo, ¿son las organizaciones responsables de sus resultados u outputs cuya mejora persiguen? Esto último, desde luego, implica un nivel expansivo de control exógeno a la misma organización. Sin embargo, como indica un experto en el terreno de la medida del funcionamiento «los outputs no son verdaderas medidas del funcionamiento; los resultados sí lo son... por tanto, confundir outputs con resultados es tomar equivocadamente la actividad por el logro» (HATRY, 1999: ix).

${ }^{16}$ Por ejemplo, en algunos sistemas los gestores tenían más poder discrecional y estaban sujetos a menos reglas y limitaciones administrativas (un enfoque de «dejar que los gestores gestionen») mientras que en otros sistemas los gestores estaban sujetos a limitaciones y criterios de mercado (un enfoque de «hacer que los gestores gestionen»). Obviamente, hay aquí dos teorías muy diferentes. Una de ellas considera las limitaciones burocráticas como impedimentos para que surja la iniciativa y la invención; la otra ve en las limitaciones de mercado y la disciplina el motor para un mejor funcionamiento. En relación con esto véase KATTL (1997).

\section{Bibliografía}

ABErbaCH, Joel D. (1990), Keeping A Watchful Eye: The Politics of Congressional Oversight, Washington: The Brookings Institution.

Aberbach, Joel D. (1990); Robert, D.; Putnam, y Rockman, Bert A. (1981), Bureaucrats and Politicians in Western Democracies, Combridge: Harvard University Press.

ABerbach, Joel D. (1990), y Rockman, Bert A. (2000), In the Web of Politics: Three Decades of the U.A. Federal Executive. Washington: The Brookings Institution Press.

AlCHiAN, Armen A. (1950), «Uncertainty, Evalution, and Economic Theory», Journal of Political Economy, 58: 211.221.

ALCHIAN, Armen A. (1950), y DemseTz, Harold (1972), «Production, Information Costs, and Economic Organization», American Economic Review, 62: 777-795.

BENDOR, Jonathan (1994), «The Fields of Bureaucracy and Public Administration: Basic and Applied Research», Joumal of Public Administration Research an Theory, 4: 27-39.

BENDOR, Jonathan (1994), y HAMmOND, Thomas (1992), «Rethinking Allison's Models», American Political Science Review, 86: 301-322.

BENDOR, Jonathan (1994); MoE, Terry M., y ScHotTs, Kenneth (2000), «Recycling the Garbage Can: An Assessnebt of the Research Program», American Political Science Review, 94.

Bendor, Jonathan (1994); TAYlor, Serge, y VAN GaAlen, Roland, «Politicians, Bureaucrats, and Asymmetric Information», American Joumal of Political Science, 31: 796.828.

Birnbaum, Pierre (1982), The Heights of Power: An Essay on the Power Elite in France, traducido por Arthur Goldhammer, Chicago: University of Pittsburgh Press.
BlaIs, André, y Dion, Stéphane (1991), The Budget-Maximizing Bureaucrat: Appraisals y Evidence, Pittsburgh: University of Pittsburgh Press.

CAmpBell, Colin (1983), Governments Under Stress: Political Executives and Key Bureaucrats in Washington, London, and Ottawa, Toronto: University of Toronto Press.

CAmpbell, Colin (1983) y SzablowskI, George (1979), The Superbureaucrats: Structure and Behavioour in Central Agencies, Toronto: MacMillan of Canada.

Campbell, Colin (1983) y Wilson, Graham K. (1995), The Ens of Whitehall: Death of a Paradigm?, Oxford: Oxford University Press.

Carpenter, Daniel P. (1996), «Adaptive Signal Processing, Hierarchy, and Budgetary Control in Federal Regulation», American Political Science Review, 90: 283-302.

COASE, R. H. (1988), The Firm, the Market and the Law, Chicago: University of Chicago Press.

Conen, Michael D., y Axelrod, Robert (1984), «Cfoping with Complexity: The Adaptative Value of Changing Utility», American Economic Review, 74: 30-42.

Cohen, Michael D.; MARCh, James G., y Olsen, Johan P. (1972), «A Garbage Can Model of Organizational Choice», Administrative Science Quarterly, 17: 1-25.

Dogan, Mattei (1975), The Mandarins of Western Europe: The Political role of Top Civil Servants, New York: Halster Press.

FIORINA, Morris P. (1978), Congress: Keystone of the Washington Establishment, New Haven: Yale University Press.

GreEn, Donald P., y Shapiro, Ian (1994), Patbologies of Rational Choice Theory: A Critique of Applications in Political Science, New Haven: Yale University Press. 
GuLICK, Luther (1937), «Notes on the Theory of Organization -with Special reference to Government in the United States», en L. GULICK y L. URWICK (eds.), Papers onthe Science of Administration, New York: Columbia University.

Hhlperin, Morton H., y KanTer, Arnold (1973), «The Bureaucratics Perspective: A Preliminary Framework», en M. H. HALPERIN y A. KANTER (eds.), Readings in American Foreing Policy: A Bureaucratic Perspective, Boston: Little, Brown.

HATRY, Harry P. (1999), Performance Measurement: Getting results, Washington: Urban Institute Press.

Hodge, Graeme A. (1999), Privatization: An International Review of Performance, Boulder, CO: Westview Press.

Jones, Bryan D. (1994a), «A Change of Mind or a Change of Focus? A theory of Choice Reversals in Politics», Journal of Public Adminiustration Research and Theory, 4: 141-178

- (1994b), Reconceibing Decision-Making in Democratic Politics: Attention, Choice, and Public Policy, Chicago: University of Chicago Press

KETTL, Donald F. (1993), «Public Administration: The State of the Field», en Ada Finifter (ed.), Political Science: The State of the Discipline, Washington: American Political Science Association.

KETTL, Donald F. (1997), «The Global Revolution in Public Management: Driving Themes, Missing Links», Journal of Policy Analisis and Management, 16: 446-462.

KOH, B. C. (1989), Japan's Administrative Elite, Berkeley y Los Ángeles: University of California Press.

Krause, George A. (1999), A two-Way Street: The Institutional Dynamics of the Modern Administrative Sate, Pittsburgh: University of Pittsburgh Press.

KuвотA, Akira (1969), Higher Civil Servants in Postwar Japan: Their social Origins, Backgrounds, and Career Patterns, Princeton, NJ: Princeton University Press.

KuHN, Thomas S. (1996), The Structure of Scientific Revolutions, $3^{\mathrm{RD}}$, Chicago: University of Chicago Press.

LARKEY, Patrick D.; Stolp, Chandler, y WINER, Mark (1984), «Why Does Government Grow?, en Trudi C. Miller (ed.), Public Sector Performance: A Conceptual Turning Point, Baltimore: Johns Hopkins University Press.

LAVER, Michael, y SHEPSLE, Kenneth A. (1994), «Cabinet Government in Theoretical Perspective», en Michael Laver y Kenneth A. SHePsle (eds.), Cabinet Ministers and parliamentary Government, Cambridge, UK: Cambridge University Press.

LiGHT, Paul C. (1998), The Tides of Reform: Making Government Work, 1945-1995, New Haven: Yale University Press.

MARCH, James G. (1978), «Bounded Rationality, Ambiguity, and the Engineering of Choice», The Bell Journal of Economics, 9: 587-608.

MARCH, James, G. (1978), y Olsen, Johan P. (1983), «Organizing Political Life: What Administrative Reorganization Tells Us about Government», American Political Science Review, 77: 281-296.

- (1989), Rediscovering Institutions: The Organizational Basis of Politics, New York: The Free Press.

- (1995), Democratic Governance, New York: The Free Press

March, James G. (1995), y Cohen, Michael D. (1976), Amgiguity and Choice in Organizations, Oslo: Universitetsforlaget.

McCubBins, Mathew D. (1985), «The Legislative Desiong of Regulatory Structure», American Journal of Political Science, 29: 721-748.

MCCubBINs, Mathew D. (1985), y NoBLE, Gregory W. (1995), «The Appearance of Power: Legislators, Bureaucrats, and the Budget Process in the United States in the United States and Japan», En Peter F. CowrteY y Mathew D. McCubBins (eds.), Structure and Policy in Japan and the Utited States, Cambridge: Cambridge University Press.

McCubBins, Mathew D. (1985), Noll, Roger, y WeINGAST, Barry (1990), «Administrative Procedures as Instruments of Political Control», Journal of Law, Economics, and Organization, 6:1-20

MILLER, Gary J. (1992), Managerial Dilemmas: The Political Economy of Hierarcby, Cambridge: Cambridge University Press.

Miller, Gary J. (1992), y Hammond, Thomas H. (1994), «Why Politics is More Fundamental than Economics: Incentive-Compatible Mechanisms are Not Credible», Journal of Theoretical Politics, 6: 5-26.

Miller, Trudi C. (1984), «Conclusion: A Desing Science Perspective», en Trud C. Miller (ed.), Public Sector Performance: A Conceptual Turning Point, Baltimore: Johns Hopkins University Press.

Moe, Terry M. (1985), «The Politicized Presidency», en John E. ChubB y Paul E. Peterson (eds.), The New Direction in American Politics, Washington: The Brookings Institution

- (1989), «The politics of Bureaucratic Structure», en John E. CHUBB y Paul E. PEterson (eds.), Can the Government Govern?, Washington: The Brookings Institution.

- (1990), «Political Institutions: The Neglected Side of the Story», Journal of Law, Economics, and Organization, 6: 213-253.

- (1993), «Presidents, Institutions, and Theory», en George C. Edwards III, John H. Kessel y Bert A. Rockman (eds.), Researching the Presidency: Vital Questions, New Approaches, Pittsburgh: University of Pittsburgh Press.

- (1994), «Integrating Politics and Organizations: Positive Theory and Public Administration», Journal of Public Administration Research and Theory, 4: 17-25.

Moe, Terry M., y CALDWELL, Michael (1994), «The Institutional Foundations of Democratic Government: A Comparison of Presidential and Parliamentary Systems», Journal of Institutional and Theoretical Economic, 150: 171-195.

Moe, Terry M. (1994), y Howell, William G. (1999), «The Presidential Power of Unilateral Action», Journal of Law, Economics, and Organization, 15: 132-179.

MotT, Paul E. (1972), The Characteristics of Effective Organizations, New York Harper \& Row

NISKANEN, William A. (1971), Bureaucracy and Representative Government, Chicago: Aldine-Atherton.

- (1991), «A Reflection on Bureaucracy and Representative Government», en A Bass y S. Dion (eds.), The Budget-Maximizing Bureaucrat: Evidence and Appraisals, Pittsburgh: University of Pittsburgh Press.

Olsen, Johan P. (1983), Organized Democracy: Political Institutions in a Welfasr State - The Case of Norway, Oslo: Universitetsforlaget.

Osborne, David, y Gaebler, Ted (1993), Reinventing Government: How the Entrepreneurial Spirit Is Transforming the Public Sector, New York: Plume.

PAGE, Edward C., y WRIGHT, Vincent (1999), Bureaucratic Elites in Western European States: A Contemporary Analysis of Top Officials, Oxford: Oxford University Press.

PIerson, Paul (2000), «Increasing Returns, Path Dependence, and the Study of Politics», American Political Science Review, 94: 251-268.

SHEPSLE, Kenneth A. (1986), «Institutional Equilibrium and Equilibrium Institutions», en Herbert F. Weisberg (ed.), Political Science: The Science of Politics, New York: Agathon Press.

- (1989), «Studying Institutions: Some Lessons from the Rational Choice Approach», Joumal of Theoretical Politics, 1: 131-147. 
SHEPSLE, Kenneth A., y WEINGAST, Barry R. (1987), «The Institutional Foundations of Committee Power», American Political Science Review, 81: 85-104.

Simon, Herbert A. (1975) (1947), Administrative Bebavior: A Study of Decision-Making Processes in Administrative Organization, $3^{\text {rd }}$ ed., New York: The Free Press.

Simon, Herbert A. (1958), "The Decision-Making Schema": A Reply», Public Administration Review, 18: 60-63.

Suleiman, Ezra S. (1974), Politics, Power, and Bureaucracy in France, Princeton, NJ: Princeton University Press.

Truman, David B. (1964) (1951), The Government Process: Political Interests and Public Opinion, New York: Alfred A. Knopf.
WeingaST, Barry R., y MORAN, Mark (1983), «Bureaucratic Discretion or Congressional Control? Regulatory Policymaking by the Federal Trade Commission», Journal of Political Economy, 91: 756-800.

WildAvSKY, Aaron (1961), «Political Implications of Budgetary reform», Public Administration Review, 21: 183-190.

WildavskY, Aaron, y Hammond, Arthur (1965), «Comprehensive Versus Incremental Budgeting in the Department of Agriculture», Administrative Science Quarterly, 10: 321-346.

Wolf, Patrick J. (1999), «Neutral and Responsive Competence: The Bureau of the Budget, 1939-1948, Revisited», Administration E Society, 31: 142-167.

Wood, B. Dan, y Waterman, Richard W. (1991), «The Dynamics of Political Control of the Bureaucracy», American Political Science Review, 85: 801-828. 


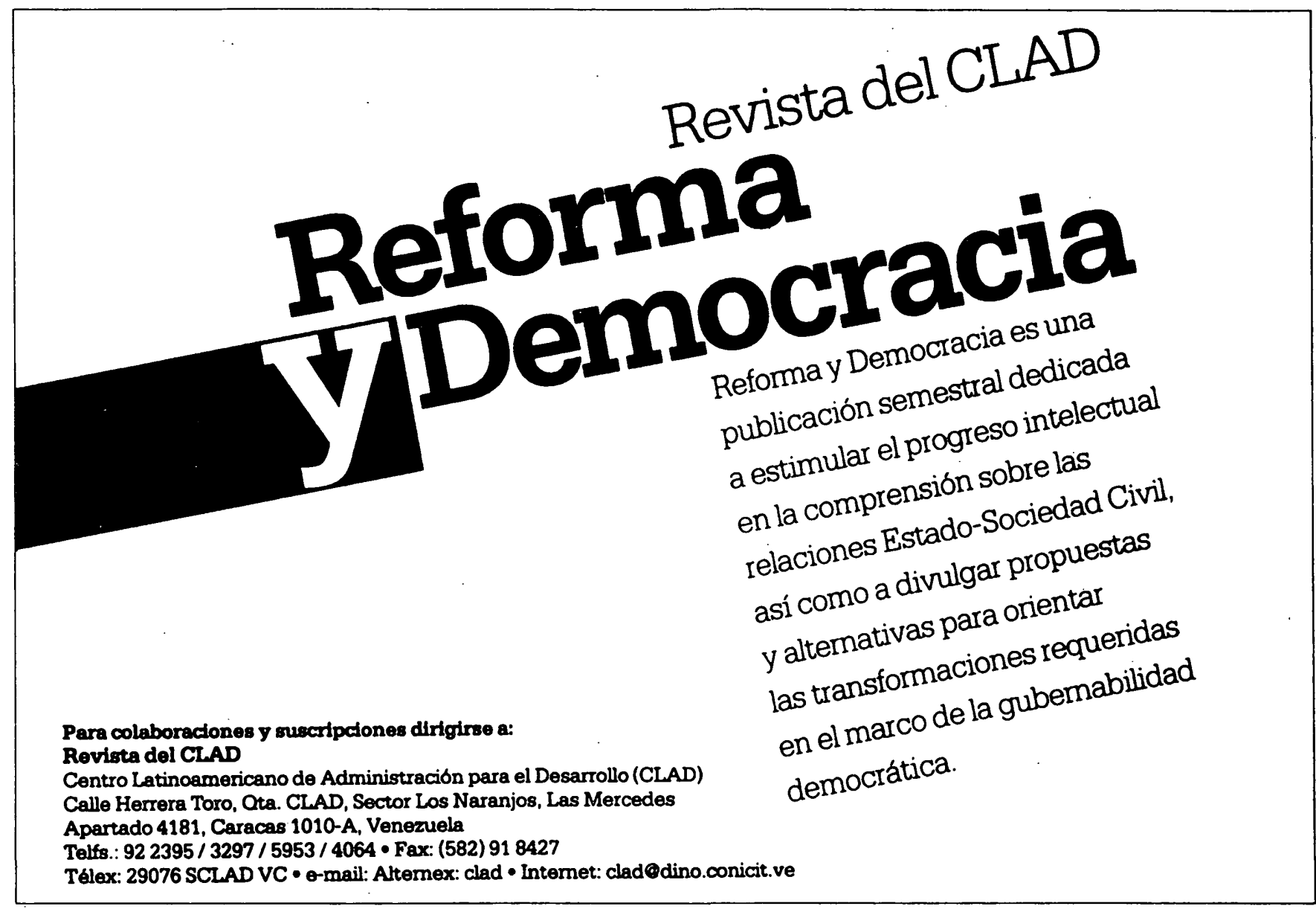

